Eur. J. Clin. Chem. Clin. Biochem.

Vol. 32, 1994, pp. 873-880

() 1994 Walter de Gruyter \& Co. Berlin - New York

\title{
Fitting Response Curves for Radioimmunoassays or Immunoradiometric Assays
}

\author{
By H.-P. Altenburg
}

Med. Statistik, Biomathematik und Informationsverarbeitung, Fakultät für Klinische Medizin Mannheim der Universität Heidelberg, Heidelberg, Germany

(Received February 9. 1993/May 2/July 29, 1994)

Summary: The paper describes non-linear regression methods for the evaluation of radioimmunoassay or immunoradiometric assay data. The underlying model is an overdispersed Poisson process with four regression line parameters and one parameter related to the overdispersion of the variance. A generalized least-squares algorithm is described for the parameter estimation of non-contaminated data. In the presence of outliers in Y-direction, the results are improved by a winsorized version of the generalized least-squares method.

\section{Introduction}

Radioimmunoassays or immunoradiometric assays are now standard procedures for the estimation of hormones and other materials in very small concentrations. The use of natural proteins such as thyropexin (thyroid hormone binding globulin) (1) or antibodies (2) as assay reagents was described in 1960. Antigens or antibodies, labelled with radioisotopes, are incubated with the test material, and the bound or free fraction of radiation is determined. Methods using natural binding proteins (receptors) may give closer estimates of the biological activity of a hormone, but antibodies, when specific and of high titre, offer the advantage that they can be made widely available and used over extended periods. Since radioligand assays do not depend on responses measured in living organisms, they are not strictly bioassays. Nevertheless they are so similar in structure that the following model is considered from this viewpoint.

Many users of radioimmunoassays or immunoradiometric assays make no formal statistical analyses. In view of the precision and the smoothness of the response curves, they rely on reading from freehand curves constructed from the data. In particular, they neglect the merits of simultaneous trials of standard and test preparations, statistical tests of validity and the estimation of error. Reliance on graphical methods might be satisfac- tory, if the experimentation is well controlled and the response curve appears very smooth. But for a more critical examination of the data, and the estimation of parameters according to recognized statistical principles, statistical regression procedures must be used for the analysis.

The plot of the observed counts against the logarithm of the administered dose is usually a symmetric sigmoid curve with an upper and lower asymptotic level corresponding to the bound count $\delta_{0}$ at zero dose and the non-specific count $\delta_{\infty}$ at "infinite" dose. Proposals for calculating radioimmunoassay results include empirical methods (e.g. spline function approach or point-to-point interpolation) and those in which it is assumed that the standard curve is a member of a particular family of curves. Among the second group three families of curves have been used: the logistic family, those based on the law of mass action, and linear regression approaches. In the linear regression approach functions of the observed counts such as the "fraction bound", the "fraction free", the "bound/initial bound ratio" or the "bound/free ratio" and their reciprocals are normally plotted against the dose or the logarithm of dose, and any of these relationships will show an approximately linear regression over some range of dose, but will al- 
most certainly be disconcertingly non-linear if the range is extended.

Also many radioimmunoassays are analysed using a linear regression analysis based on the logit-log transformation of the data. For this transformation the counting data must represent the percentage of specifically bound material, i.e., the non-specific binding must be subtracted from the counting data, which must then be expressed as a percentage of the control adjusted for non-specific binding. Several problems arise in this type of analysis. First, it is assumed that the control and nonspecific binding data are known exactly, when in fact they are also subject to experimental error. Essentially, this means that the endpoints of the curve are being fixed. Second, the observed dose-response data sometimes appear outside these fixed asymptotic endpoints (e.g. some observations are less than the non-specific binding value). The logit-log analysis cannot handle data like this and these data points can only be removed from the analysis.

A mathematical model representing the statistical features of experimental data should therefore concentrate attention on the original observations rather than on derived functions. This also allows the fitting of control and non-specific binding levels. Additionally, data do not have to be discarded if they fall outside an appropriate range. Raab (3) and Finney (4) have pointed out that the mass-action law approach can assume only a very limited range of shapes, and cannot represent assays where the standard curve slope is less than a specified value. However, a four- or five-parameter logistic model will fit data at least as well over a wide range of doses in many but not all circumstances, particularly when both the binding site concentration and the equilibrium constant are small.

Because radioligand assays are based upon records of radiation counts in a fixed time at various doses, a quite realistic assumption concerning the distribution of counts is that they are realisations of some stochastic Poisson process. Based on an overdispersed Poisson model, one can express the expectation of the observed counts by a four-parametric non-linear function. In contrast to the normal Poisson model, the marginal variance depends on a further single parameter which additionally has to be estimated. In the normal case, when the data are not contaminated, a generalized least squares technique can be used to estimate the parameters. Nevertheless, this type of model does not provide a good fit for all experimental situations. The following sections therefore describe the underlying mathematical model and give an improved version of the estimation algorithm - a generalized least squares algorithm - as presented by Altenburg \& Rosenkranz (5). But, since radio- immunoassay data often show outliers in Y-direction, one has to use a robust estimation procedure to minimize the influence of the outliers on the estimates. Different robust estimation procedures have been compared by $\mathrm{Al}$ tenburg (6). Finally, therefore, a generalized least squares algorithm is presented, which the author regards as the most robust version of the generalized least squares technique. The aim of the paper is not only to present estimation procedures for the single dose response calibration, but also to give a procedure that can simultaneously estimate several dose response curves in different experimental situations, and which furthermore can be easily implemented on a standard statistical package.

\section{The Underlying Model}

Normally the expectation of the count (fixed time) in a radiometric experiment is given by a four-parameter sigmoid function:

$$
\begin{aligned}
\lambda= & \mathrm{E}[\text { count } \mid \text { dose }=x]= \\
& \delta_{0}+\rho G\left(\theta_{0}+\theta_{1} \log x\right),
\end{aligned}
$$

where $x$ denotes the ligand concentration or the administered dose, $\rho=\delta_{\infty}-\delta_{0}$ is the range of counts, $\theta_{0}$ and $\theta_{1}$, are curve parameters (real numbers): the concentration at which we observe the $50 \%$ effect (EC 50), and the slope of the curve, respectively. $G(z)$ is a function of the administered dose $z$ that changes smoothly from 0 to 1 as $z$ tends to $+\infty$. Immunoradiometric assay curves differ from radioimmunoassay curves, in that $G(z)$ becomes $G^{*}(z)=1-G(z)$.

The monotone increasing function $G\left(\theta_{0}+\theta_{1} \log x\right)$ corresponds in the four-parameter logistic curve, and in the radioimmunoassay case to the logistic function

$$
\begin{aligned}
& G\left(\theta_{0}+\theta_{1} \log x\right)= \\
& \begin{cases}1-1 /\left(1+\exp \left(-\left(\theta_{0}+\theta_{1} \log x\right)\right)\right) & \text { if } x>0 \\
1 & \text { if } x=0\end{cases}
\end{aligned}
$$

which is called the Logrt model. As in a quantal bioassay, we can allow other distribution functions, such as e.g. the PROBIT model

$$
\begin{aligned}
& G\left(\theta_{0}+\theta_{1} \log x\right)= \\
& \begin{cases}1-\Phi\left(\theta_{0}+\theta_{1} \log x\right) & \text { if } x>0 \\
1 & \text { if } x=0\end{cases}
\end{aligned}
$$

where $\Phi(u)$ is the distribution function of the standard normal distribution, or in more special scientific applica= tions the WeIBULl modell 


$$
\begin{aligned}
& G\left(\theta_{0}+\theta_{1} \log x\right)= \\
& \begin{cases}1-\exp \left(-\exp \left(-\left(\theta_{0}+\theta_{1} \log x\right)\right)\right) & \text { if } x>0 \\
1 & \text { if } x=0\end{cases}
\end{aligned}
$$

Generally, no single fixed distribution will fit all situations. As Ratkowsky \& Reedy (7) pointed out, the parametrization presented in Eq. 1 is the best one under "close to linear" properties.

In the following we look only at a single treatment group experiment, with dose levels $x_{1}, \ldots, x_{d}$ and parameter vector $\Theta=\left(\delta_{0}, \rho, \theta_{0}, \theta_{1}\right)$ to be estimated. If there were no measurement errors in the process of recording the counts, maximum likelihood theory could be applied for parameter estimation. Unfortunately measurement errors occur and the number of recorded counts is determined by the statistical nature of radioactive decay and additional random erorrs. Thus the variance is expected to be larger than the variance obtained from a Poisson process alone.

In most applications one can suppose that the relative measurement error is independent of the recorded counts. Hence, assuming that the number of counts $y_{i}$ at concentration $x_{i}$ conditional on the errors $e_{i}$ form a sequence of independent random variables, then expectation and variance are given by

$$
\begin{aligned}
& \mathrm{E}\left[y_{i} \mid \Theta, e_{i}\right]= \\
& \quad \operatorname{Var}\left[y_{i} \mid \Theta, e_{i}\right]=\left(\delta_{0}+\rho G\left(\theta_{0}+\theta_{1} \log x\right)\right) e_{i},
\end{aligned}
$$

$$
\begin{aligned}
& \mathrm{E}\left[e_{i}\right]=1, \\
& \quad \operatorname{Var}\left[e_{i}\right]=\kappa^{2} \quad e_{i} \text {, uncorrelated }
\end{aligned}
$$

for every $i=1, \ldots, d$. The marginal moments of $y_{i}$ then have the form

$$
\begin{aligned}
& \lambda_{i}^{\Theta}=E\left[y_{i} \mid \Theta\right]=\delta_{0}+\rho G\left(\theta_{0}+\theta_{1} \log x\right) \\
& \Lambda_{i}^{\Theta}=\operatorname{Var}\left[y_{i} \mid \Theta\right]=\lambda_{i}^{\Theta}\left(1+\kappa^{2} \lambda_{i}^{\Theta}\right),
\end{aligned}
$$

where the variance contains an additional parameter $\kappa$.

\section{The Estimation Procedure}

Because we are not able to model the distribution of counts but only its first and second moment, maximum likelihood estimates are not available. However, quasilikelihood methods (cf. Wedderburn (8) or McCullagh (9)) imply that an estimate $\hat{\Theta}$ of $\Theta$ is a root of the equations

$$
\sum_{i=1}^{\mathrm{d}} \frac{\partial \lambda_{i}^{\Theta}}{\partial \Theta_{j}} \frac{y_{i}-\lambda_{i}^{\Theta}}{\Lambda_{i}^{\Theta}}=0, \quad j=1, \ldots, 4,
$$

where $\frac{\partial}{\partial \Theta_{j}}$ denotes the partial derivation to the $j$-th component of $\Theta$ (e.g. $\frac{\partial}{\partial \Theta_{1}} \equiv \frac{\partial}{\partial \delta_{0}}$ ). Quasi-likelihood estimators have similar properties as maximum likelihood estimators but in those cases where maximum likelihood estimators exist they are less efficient.

To get the quasi-likelihood estimators, we can use the non-linear estimation procedure of a statistical package like the SAS procedure NLIN, minimizing the function

$$
S(\Theta)=\sum_{i=1}^{d} w_{i}\left(y_{i}-\lambda_{i}^{\Theta}\right)^{2}
$$

with the weights

$$
w_{i}=\frac{1}{\lambda_{i}^{\Theta}\left(1+\kappa^{2} \lambda_{i}^{\Theta}\right)}, \quad \text { for } i=1, \ldots, d
$$

In terms of both point estimation, confidence interval coverage and a computational point of view, Beal \& Sheiner (10) found that a generalized least squares technique is one of the test tractable estimating procedure for a heteroscedastic problem of the present type. Starting values for the parameters in step 1 and 2 of the estimation algorithm are obtained as follows.

Step 0

Zero might be an adequate starting value for all the curve parameters, $\theta_{0}, \theta_{1}$ and the variance parameter $\kappa$. The asymptotic level values $\hat{\delta}_{0}$ and $\hat{\delta}_{\infty}$ are easily estimated from the mean or median values of $y_{i}$, at the nonspecific (lowest) or asymptotic (highest) dose levels $x_{1}$ or $x_{d}$, respectively, from which we can compute the starting value for $\rho$ by

$$
\hat{\rho}=\hat{\delta}_{\infty}-\hat{\delta}_{0} \text {. }
$$

In case of an immunoradiometric assay the roles of $\hat{\delta}_{0}$ and $\hat{\delta}_{\infty}$ have to be interchanged.

Then the estimators $\hat{\Theta}$ can be obtained by the following algorithm steps $\left(\hat{\kappa}^{(j)}\right.$ and $\hat{\Theta}^{(j)}$ denote the estimates in iteration step $j$ ):

Step 1

Ordinary least squares estimator of $\Theta$ : Using the above starting values calculate $\hat{\Theta}^{(0)}$ by minimizing the expression

$$
\sum_{i=1}^{d}\left(y_{i}-\lambda_{i}^{\Theta}\right)^{2}
$$

Iterate the following two steps for $j=1,2, \ldots$ :

Step 2

Given $\hat{\Theta}^{(j-1)}$ and $\hat{\kappa}^{(j-1)}$ we can obtain the estimator $\hat{\kappa}^{(j)}$ of $\kappa$ by minimizing (with respect to $\kappa$ ): 


$$
\sum_{i=1}^{d}\left(r_{i}^{2}-\Lambda_{i}^{\hat{\Theta}^{(j-1)} \cdot \hat{k}^{(j-1)}}\right)^{2} /\left(\Lambda_{i}^{\hat{\Theta}^{(j-1)} \cdot \hat{K}^{(j-1)}}\right)^{2},
$$

where $r_{i}=y_{i}-\lambda_{i}^{\dot{\Theta}^{(j-1)}}$ are the residuals of step $j-1$ and $\Lambda_{i}^{\hat{\theta}}$ is according to Eq. 8 .

Step 3

Weighted least squares estimator of $\Theta$ given $\hat{\kappa}^{(i-1)}$ and $\hat{\Theta}^{(j-1)}$ : Calculate $\hat{\Theta}^{(j)}$ such that

$$
\sum_{i=1}^{d}\left(y_{i}-\lambda_{i}^{\hat{\Theta}}\right)^{2} / \Lambda_{i}^{\hat{\Theta}, \hat{\kappa}}=\min .
$$

Step 2 and Step 3 have to be iterated at least two times to eliminate the effect of the inefficient unweighted least-squares estimate $\Theta^{(0)}$. The iteration ends if the change of the estimate $\hat{\kappa}$ of the variance parameter is less than a pregiven value $\varepsilon$. In our test data examples we found that normally four or five iteration cycles seem to be sufficient for the estimation of the parameters of one or two groups. In applications with more than two groups the number of iterations has to be slightly increased. The numerical calculations involved in the three steps of the algorithm can be done by the computational methods of Marquardt (in Step 1 and 3) or GaussNewton (in Step 2).

\section{A Non-Linear Robust Regression Method}

Normally the technique of radioimmunoassay is procedurally complicated and it is not unusual that outliers appear in the data. In the case of non-linear regression the development of robust estimation procedures is not free from troubles and consumes a lot of computing time. In standard applications of the radioimmunoassay technique, outliers or long-tailed distributions occur only in the Y-direction, so it is assumed that estimation procedures for M-estimates such as Huber type estimation procedures deal adequately with outliers. As shown in 1. c. (6) the best robust method for estimating radioimmunoassay or immunoradiometric data according to the above described overdispersed Poisson model is the following procedure. The procedure uses a metrical winsorization of the data as described in Huber (11).

\section{Winsorized Generalized Least Squares Algorithm}

Step 1

Start using the generalized least squares algorithm as . described in Section 2.

\section{Step 2}

Winsorize metrically the original observations $y_{i}$ and replace them in the following way be pseudo-observations $y_{i}^{*}$ :

$$
y_{i}^{*}= \begin{cases}y_{i} & \text { if }\left|r_{i}\right| \leq k s_{i} \\ \hat{y}_{i}+\operatorname{sign}\left(r_{i}\right) k s_{i} & \text {, } \text { otherwise }\end{cases}
$$

where $r_{i}=y_{i}-\hat{y}_{i}$ are the residuals, $s_{i}$ are the actual estimates of the standard error of the residuals $r_{i}$, and $\hat{y}_{i}$ are the last fitted values of $y_{i}$. The constant $k$ denotes a tuning constant regulating the amount of robustness.

\section{Step 3}

On the basis of the winsorized data set, estimate $\kappa$ and $\Theta$ by the procedure-Steps 2 and 3 of the generalized least squares algorithm in Section 2.

Iterate Step 2 and 3 again until all observations or pseudo-observations remain unchanged by Eq. 16 . The procedure converges and the result is a $\mathrm{M}$-estimator. We found that one needs about 22 iteration cycles of Step 2 and 3 to get appropriate estimates. In our opinion good choices for the tuning constant $k$ are values with $1 \leq k \leq 2$, e.g. $k \approx 1.6$. The contamination of the data set should not be too high (at most $20 \%$ ). The winsorization procedure fails if outliers exist in both $\mathrm{X}$-direction and Y-direction. Then a probabilistic technique like a non-linear generalization of the least median of squares methods might be appropriate. Although the probabilistic least median of squares procedure or related techniques are formally easily extended to the non-linear regression case, the computation of these estimators is far from trivial.

\section{Numerical Illustration and Discussion}

An example is now presented to illustrate the above algorithms. The data set given in table 1 is from Tiede \& Pagano (12) representing thyrotropin standards. The dose is measured in mIU/l. The outlier in this data set is marked by an asterix. Instead of a zero dose in the original data set we use a value of 0.01 , because a value of zero leads to an invalid expression in the term $\log ($ dose $)$ of the underlying model. In table 2 the estimation results for this data set are given. Listed are the estimates for

Tab. 1 Data set of Tiede \& Pagano (12).

\begin{tabular}{llllllll}
$\begin{array}{l}\text { Thyrotropin } \\
\text { dose } \\
\text { (mIU/l) }\end{array}$ & 0 & 2 & 5 & $10^{\circ}$ & 20 & 50 & 100 \\
\hline $\begin{array}{l}\text { Radio- } \\
\begin{array}{l}\text { activity } \\
\text { (counts/s) }\end{array}\end{array}$ & 7720 & 6664 & 4994 & 3410 & $4478^{*}$ & 1302 & 1025 \\
\hline & 6804 & 4948 & 3208 & 2396 & 1377 & 1096 \\
\hline
\end{tabular}


Tab. 2 Parameter estimates for the data set of Tiede \& Pagano (12) (tab. 1) for the probit model with $\log _{10}($ dose), using different

estimation procedures (OLS $=$ ordinary least squares, GLS $=$ generalized least squares).

\begin{tabular}{llllllrr}
\hline Procedure & $\delta_{0}$ & & $\theta_{1}$ & $0_{0}$ & $\kappa$ & SAE & \multicolumn{1}{c}{ MSE } \\
\hline OLS & 7967.02 & -6947.08 & -1.2731 & 1.5207 & - & 347.5 & 425709 \\
OLS no outlier & 7928.01 & -6851.40 & -1.4986 & 1.9084 & - & 87.6 & 20117 \\
GLS & 7728.06 & -6705.25 & -1.5082 & 1.6908 & 0.04161 & 350.1 & 451207 \\
GLS no outlier & 7949.59 & -6894.66 & -1.4533 & 1.8521 & 0.0007957 & 89.9 & 20922 \\
GLS winsorized & 7938.36 & -6890.50 & -1.4457 & 1.8204 & 0.0007854 & 92.0 & 18910 \\
\hline
\end{tabular}

Tab. 3 Estimated counts and effective concentrations with $95 \%$ confidence limits for the iterated and winsorized generalized least squares algorithm.

Data Set: Tiede \& Pagano (12) (tab. 1)

\begin{tabular}{|c|c|c|c|c|c|c|c|c|}
\hline \multirow[t]{2}{*}{$\eta$} & \multicolumn{4}{|c|}{ Iterated generalized least squares } & \multicolumn{4}{|c|}{ Winsorized generalized least squares } \\
\hline & $\begin{array}{l}\text { Estimated } \\
\text { counts }\end{array}$ & $\begin{array}{l}\text { Concen- } \\
\text { tration } \\
\mathrm{EC} \eta\end{array}$ & $\begin{array}{l}\text { Lower } \\
95 \% \\
\text { confidence } \\
\text { limit }\end{array}$ & $\begin{array}{l}\text { Upper } \\
95 \% \\
\text { confidence } \\
\text { limit }\end{array}$ & $\begin{array}{l}\text { Estimated } \\
\text { counts }\end{array}$ & $\begin{array}{l}\text { Concen- } \\
\text { tration } \\
E C \eta\end{array}$ & $\begin{array}{l}\text { Lower } \\
95 \% \\
\text { confidence } \\
\text { limit }\end{array}$ & $\begin{array}{l}\text { Upper } \\
95 \% \\
\text { confidence } \\
\text { limit }\end{array}$ \\
\hline 1 & 7661.00 & 0.328 & 0.0320 & 3.361 & 7869.46 & 0.328 & 0.2303 & 0.468 \\
\hline 2 & 7593.95 & 0.476 & 0.0560 & 4.042 & 7800.55 & 0.463 & 0.3349 & 0.641 \\
\hline 3 & 7526.90 & 0.602 & 0.0797 & 4.546 & 7731.65 & 0.577 & 0.4246 & 0.783 \\
\hline 4 & 7459.85 & 0.719 & 0.1040 & 4.968 & 7662.74 & 0.680 & 0.5075 & 0.911 \\
\hline 5 & 7392.79 & 0.830 & 0.1290 & 5.432 & 7593.84 & 0.777 & 0.5868 & 1.030 \\
\hline 10 & 7057.53 & 1.362 & 0.2699 & 6.869 & 7249.31 & 1.231 & 0.9653 & 1.569 \\
\hline 15 & 6722.27 & 1.901 & 0.4428 & 8.163 & 6904.79 & 1.678 & 1.3498 & 2.086 \\
\hline 20 & 6487.01 & 2.479 & 0.6547 & 9.384 & 6560.26 & 2.147 & 1.7611 & 2.617 \\
\hline 25 & 6051.74 & 3.112 & 0.9139 & 10.598 & 6215.74 & 2.652 & 2.2117 & 3.181 \\
\hline 30 & 5716.48 & 3.818 & 1.2304 & 11.848 & 5771.22 & 3.207 & 2.7127 & 3.791 \\
\hline 35 & 5381.22 & 4.614 & 1.6170 & 13.167 & 5526.69 & 3.824 & 3.2761 & 4.463 \\
\hline 40 & 5045.96 & 5.523 & 2.0901 & 14.592 & 5182.17 & 4.518 & 3.9166 & 5.212 \\
\hline 45 & 4710.69 & 6.571 & 2.6712 & 16.167 & 4837.64 & 5.310 & 4.6524 & 6.061 \\
\hline 50 & 4375.43 & 7.798 & 3.3882 & 17.947 & 4493.12 & 6.225 & 5.5073 & 7.037 \\
\hline 55 & 4040.17 & 9.253 & 4.2780 & 20.015 & 4148.59 & 7.298 & 6.5133 & 8.176 \\
\hline 60 & 3704.91 & 11.011 & 5.3900 & 22.493 & 3804.07 & 8.577 & 7.7147 & 9.535 \\
\hline 63 & 3503.75 & 12.253 & 6.1904 & 24.254 & 3597.35 & 9.472 & 8.5549 & 10.488 \\
\hline 65 & 3369.64 & 13.179 & 6.7901 & 25.578 & 3459.54 & 10.135 & 9.1756 & 11.194 \\
\hline 70 & 3034.38 & 15.927 & 8.5677 & 29.608 & 3115.02 & 12.084 & 10.9930 & 13.284 \\
\hline 75 & 2699.12 & 19.539 & 10.8450 & 35.202 & 2770.49 & 14.611 & 13.3236 & 16.022 \\
\hline 80 & 2363.86 & 24.533 & 13.7987 & 43.617 & 2425.97 & 18.050 & 16.4456 & 19.811 \\
\hline 85 & 2028.59 & 31.987 & 17.7226 & 57.731 & 2081.44 & 23.094 & 20.9228 & 25.490 \\
\hline 90 & 1693.33 & 44.662 & 23.2703 & 85.719 & 1736.92 & 31.488 & 28.1615 & 35.208 \\
\hline 95 & 1258.07 & 73.250 & 32.7175 & 163.997 & 1392.39 & 49.856 & 43.3916 & 57.284 \\
\hline 96 & 1291.01 & 84.606 & 35.7934 & 199.985 & 1323.49 & 56.998 & 49.1570 & 66.089 \\
\hline 97 & 1223.96 & 101.007 & 39.8153 & 256.243 & 1254.58 & 67.194 & 57.2763 & 78.829 \\
\hline 98 & 1156.91 & 127.833 & 45.6265 & 358.153 & 1185.68 & 83.626 & 70.1351 & 99.712 \\
\hline 99 & 1089.86 & 185.296 & 56.0552 & 612.517 & 1116.77 & 118.056 & 96.3992 & 144.578 \\
\hline
\end{tabular}

the robust regression procedure described above under the probit model set, compared with ordinary least squares and generalized least squares with and without the outlier. As a measure of robustness of the procedure we use the sum of the absolute residuals $r_{i}$

$$
\mathrm{SAE}=\sum_{i}\left|y_{i}-\hat{y}_{i}\right| / n=\sum_{i}\left|r_{i}\right| / n
$$

and compare it with the classical mean squared error. Table 3 lists the estimated counts and effective concentration values $\mathrm{EC} \eta$ for selected values of $\eta, 1 \leq \eta \leq 99$, with the corresponding $95 \%$-confidence limits for both the generalized and the winsorized generalized least squares algorithm. The confidence limits have been computed from the final covariance matrix of the four parameter estimates by the $\delta$-method. The results in table 3 are plotted in figures 1 and 2 . We see that even a single outlier can cause very large confidence intervals. The ordinary least squares fit of this test data set shows only slightly worse estimation results than the generalized least squares algorithm, and in many dose response calibration situations the ordinary least squares approach might therefore be sufficient for the corresponding practical applications. Possibly this example is a very ex- 


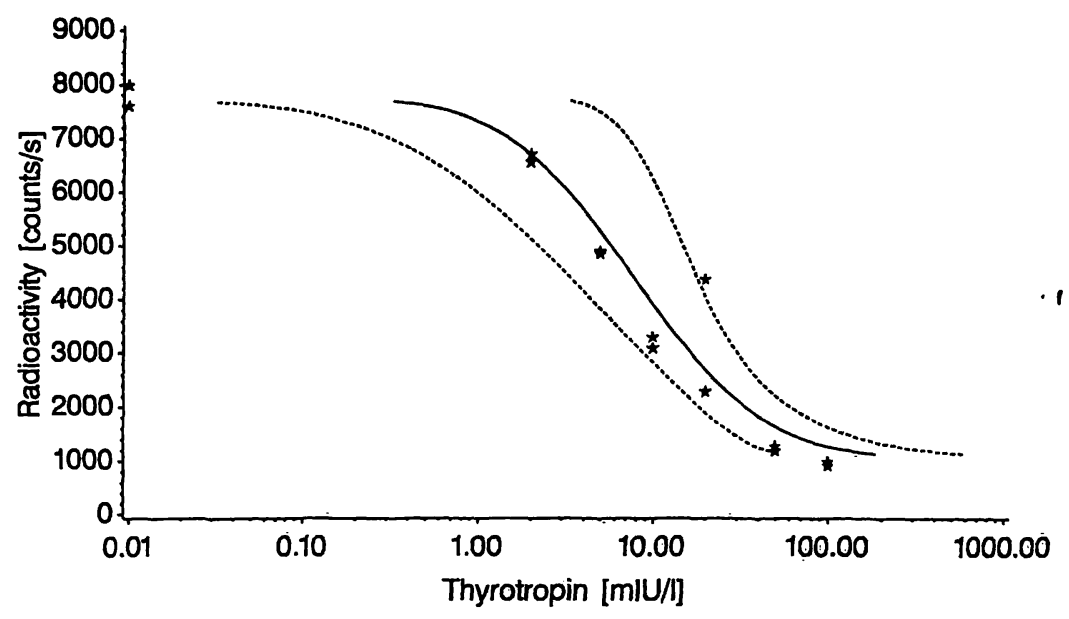

Fig. 1 Estimated concentrations, generalized least squares algorithm Probit model, dashed lines: $95 \%$ confidence limits of the thyrotropin concentrations.

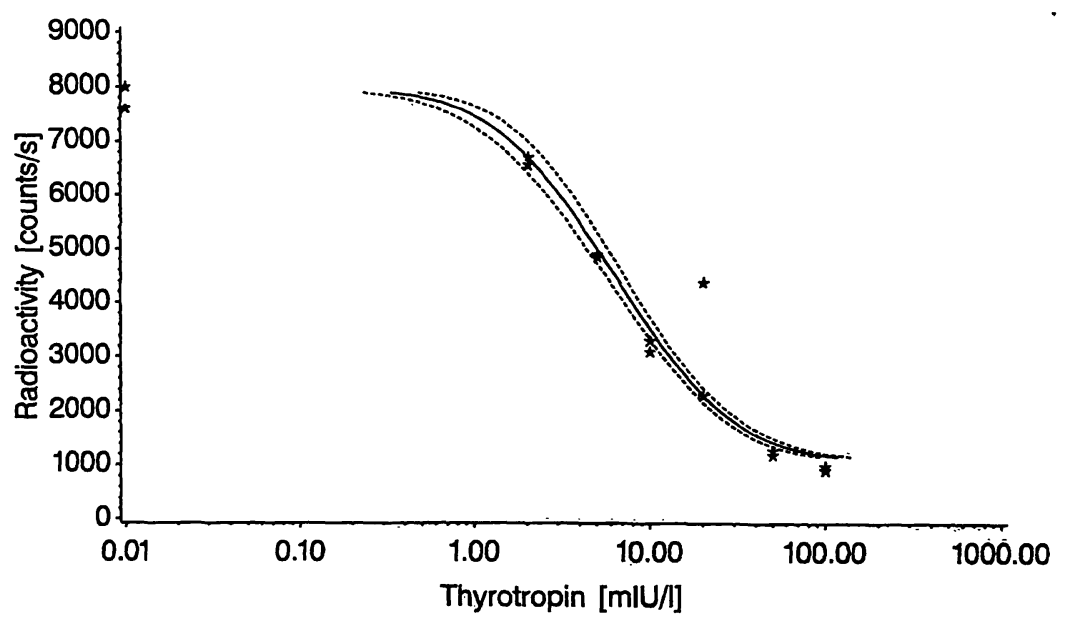

Fig. 2 Estimated concentrations, winsorized generalized least squares algorithm Probit model, dashed lines: $95 \%$ confidence limits of the thyrotropin concentrations.

treme case and "normal" outliers might lead to smaller differences in the confidence limits. The value of the tuning constant used was $k=1.6$. In the normal generalized least squares case we needed 3 iteration cycles of Step 2 and 3, whereas the winsorized generalized least squares required 22 iteration cycles until convergence.

One can imagine many similar experimental situations but one cannot test the estimation procedures in all of them. In the standard applications tested, we found that the winsorization works well if we have outliers in the Y-direction and the resulting parameter estimates are $\mathrm{M}$ estimates. The method might fail if we have outliers in both the X-and Y-directions, and in such a case the procedure may produce little improvement. This might happen if the dose values are the result of a thinning process in the experiment, where each thinning step leads to errors. The convergence of the winsorization could be very slow for an extreme data constellation. The number of iteration cycles changes, depending on the number of groups or the underlying experimental situation, such as the number of comparisons. A large number of groups increases the number of iteration cycles, whereas a parallel-line assay (as described in the following) needs fewer iterations than a general assay. The present example shows that a heteroscedastic non-linear regression procedure is not robust against outliers.

The estimation procedures described above are valid not only for the simple estimation of the parameters of a single dose response curve of the form (Eq. 1), but also in general situations like the estimation of the parameters for comparing several treatment groups or for estimating relative potencies. To illustrate this we assume in the following that two treatment groups are under study. A first step in comparing two different treatments should be to examine whether two parallel dose response lines with common asymptotic levels $\delta_{0}$ and $\delta_{\infty}$ must be considered. To test this we jointly estimate the 
six parameters $\delta_{0}, \rho, \theta_{0}, \theta_{1}, \theta_{2}, \theta_{3}$ in the following two equations:

for $x$ from treatment group 1:

$$
\lambda=\delta_{0}+\rho G\left(\theta_{0}+\theta_{1} \log x\right),
$$

for $x$ from treatment group 2:

$$
\lambda=\delta_{0}+\rho G\left(\theta_{0}+\theta_{1} \log x+\theta_{2}+\theta_{3} \log x\right),
$$

If the dose response curves are not parallel, the estimator of $\theta_{3}$ will be significantly different from zero, e.g. for an error level of $\alpha=0.05$ if $\hat{\theta}_{3} / \operatorname{Var}\left[\hat{\theta}_{3}\right] \geq 1.96$. The variance $\operatorname{Var}\left[\hat{\theta}_{3}\right]$ is obtained from the parameter estimates covariance matrix.

When the hypothesis of parallelism cannot be rejected, the data are sufficiently described by a common slope for both curves. Thus it is enough to estimate simultaneously the five parameters $\delta_{0}, \rho, \theta_{0}, \theta_{1}, \theta_{2}$ in the equations

$$
\begin{aligned}
& \lambda=\delta_{0}+\rho G\left(\theta_{0}+\theta_{1} \log x\right), \\
& \quad \text { for treatment group } 1 \\
& \lambda=\delta_{0}+\rho G\left(\theta_{2}+\theta_{1} \log x\right), \\
& \quad \text { for treatment group } 2 .
\end{aligned}
$$

Now the two treatments can be compared in terms of their relative potency $\pi$. The relative potency specifies how many more dose units in treatment one must be administered to obtain the same effect as treatment 2 . The logarithm of $\pi$ is given by

$$
\log \pi=\frac{\theta_{2}-\theta_{0}}{\theta_{1}}
$$

If the hypothesis of parallelism is rejected, six curve parameters $\delta_{0}, \rho, \theta_{0}, \theta_{1}, \theta_{2}, \theta_{3}$ have to be estimated simultaneously and no relative potency can be calculated. The equations to be estimated in a two treatment group experiment are

$$
\lambda=\delta_{0}+\rho G\left(\theta_{0}+\theta_{1} \log x\right),
$$$$
\text { for treatment group } 1
$$

$$
\begin{gathered}
\lambda=\delta_{0}+\rho G\left(\theta_{2}+\theta_{3} \log x\right), \\
\quad \text { for treatment group } 2 .
\end{gathered}
$$

The advantage of this approach is its smaller variances, thereby reducing the confidence limits for the effective concentrations. The generalization to more than two treatment groups is apparent.

Such an approach allows the parameter estimation in many different experimental situations, and the winsorized alternative of the generalized least squares algorithm is useful if we have outliers and do not want to repeat the measurement. Nevertheless the proposed algorithms fail if the specified model is not a correct description of the real experimental situation. In that case the final covariance matrix could be underestimated, especially in the winsorized approach, and the resulting confidence limits for the effective concentrations would be too narrow. Certainly the described model with the proposed estimation procedures does not fit all conceivable experimental situations. We can also transfer modified versions of the described procedures to non-radiometric assay methods such as ELISA experiments. But in this case an overdispersed Poisson model with the variance term given in Eq. 11 does not come into consideration.

The computations were done on a personal computer with the Statistical Analysis System. Both procedures for the parameter estimation as well as the computation of the effective concentration values and the graphics output are realized within this system as macro procedures. Especially in the case of many group comparisons the use of a fast personal computer is recommended, because the procedure otherwise consumes a lot of calculating time. For example, in the different iteration steps one has to perform the Gauss-Newton (estimation of the variance parameter $\kappa$ ) or Marquardt algorithm (estimation of the curve parameter), each needing between 12 (at the beginning) and one iteration (in the final step). The use of a fast programming language like TURBO PASCAL therefore has advantages but requires a lot of programming expenditure for the realization of many different experimental situations.

\section{References}

1. Ekins, R. P. (1960) The estimation of thyroxine in human plasma by an electrophoretic technique. Clin. Chim. Acta 5 , 453.

2. Yalow, R. S. \& Berson, S. A. (1960) Immunoassay of endogeneous plasma insulin in man. J. Clin. Invest. 39, 1157.

3. Raab, G. M. (1983) Comparison of a logistic and a mass-action curve for radioimmunoassay data. Clin. Chem. 29, 18581761.

4. Finney, D. J. (1983) Response curves for radioimmunoassay. Clin. Chem. 29, 1762-1766.
5. Altenburg, H.-P. \& Rosenkranz, G. (1989) Analysis of Dose Response Data. Heft 8 Schriftenreihe Medizinische Statistik, Biomathematik und Informationsverarbeitung. Mannheim.

6. Altenburg, H.-P. (1992) Estimation of radioimmunoassay data using robust nonlinear regression methods. In: Computational Statistics I (Dodge, Y. \& Whittaker, J., eds.) pp. 367-372, Physica-Verlag, Heidelberg.

7. Ratkowsky, D. A. \& Reedy, T. J. (1986) Choosing near-linear parameter in the four-parameter logistic model for radioligand and related assays. Biometrics $42,575-582$. 
8. Wedderburn, R. W. M. (1974) Quasi-likelihood functions, generalized linear models and the Gauss-Newton method. Biometrika 61, 439-447.

9. McCullagh, P. (1983) Quasi-likelihood functions. Annals of Statistics 11, 59-67.

10. Beal, S. L. \& Sheiner, L. B. (1988) Heteroscedastic nonlinear regression. Technometrics $30,327-338$.

11. Huber, P. J. (1981) Robust Statistics. John Wiley \& Sons, New York.
12. Tiede, J. J. \& Pagano, M. (1979) The application of robust calibration to radioimmunoasșay. Biometrics $35,567-574$.

Dr. Hans-Peter Altenburg

Fakultät für Klinische Medizin Mannheim

Med. Statistik, Biomathematik

und Informationsverarbeituing

Theodor-Kutzer-Ufer

D-68135 Mannheim :

Germany 